\title{
Molecular and cellular evidence for biased mitotic gene conversion in hybrid scallop
}

Shi Wang ${ }^{1}$, Lingling Zhang ${ }^{1}$, Jingjie $\mathrm{Hu}^{1}$, Zhenmin Bao ${ }^{1^{*}}$, Zhanjiang $\mathrm{Liu}^{2^{*}}$

\begin{abstract}
Background: Concerted evolution has been believed to account for homogenization of genes within multigene families. However, the exact mechanisms involved in the homogenization have been under debate. Use of interspecific hybrid system allows detection of greater level of sequence variation, and therefore, provide advantage for tracing the sequence changes. In this work, we have used an interspecific hybrid system of scallop to study the sequence homogenization processes of rRNA genes.

Results: Through the use of a hybrid scallop system (Chlamys farreri $Q \times$ Argopecten irradians $0^{\text {) }}$, here we provide solid molecular and cellular evidence for homogenization of the rDNA sequences into maternal genotypes. The ITS regions of the rDNA of the two scallop species exhibit distinct sequences and thereby restriction fragment length polymorphism (RFLP) patterns, and such a difference was exploited to follow the parental ITS contributions in the F1 hybrid during early development using PCR-RFLP. The representation of the paternal ITS decreased gradually in the hybrid during the development of the hybrid, and almost diminished at the 14th day after fertilization while the representation of the maternal ITS gradually increased. Chromosomal-specific fluorescence in situ hybridization (FISH) analysis in the hybrid revealed the presence of maternal ITS sequences on the paternal ITS-bearing chromosomes, but not vice versa. Sequence analysis of the ITS region in the hybrid not only confirmed the maternally biased conversion, but also allowed the detection of six recombinant variants in the hybrid involving short recombination regions, suggesting that site-specific recombination may be involved in the maternally biased gene conversion.

Conclusion: Taken together, these molecular and cellular evidences support rapid concerted gene evolution via maternally biased gene conversion. As such a process would lead to the expression of only one parental genotype, and have the opportunities to generate recombinant intermediates; this work may also have implications in novel hybrid zone alleles and genetic imprinting, as well as in concerted gene evolution. In the course of evolution, many species may have evolved involving some levels of hybridization, intra- or interspecific, the sex-biased sequence homogenization could have led to a greater role of one sex than the other in some species.
\end{abstract}

\section{Background}

Concerted evolution is the tendency of the different genes in a gene family or cluster to evolve in concert, resulting in sequence homogenization among the members of the family [1]. Two primary mechanisms, unequal crossover and gene conversion, were believed to function for the homogenization of multigene families

\footnotetext{
* Correspondence: zmbao@ouc.edu.cn; zliu@acesag.auburn.edu

'Key Laboratory of Marine Genetics and Breeding of Ministry of Education, Ocean University of China, Qingdao 266003, China

${ }^{2}$ The Fish Molecular Genetics and Biotechnology Laboratory, Aquatic Genomics Unit, Department of Fisheries and Allied Aquacultures and Program of Cell and Molecular Biosciences, Auburn University, Auburn, AL 36849, USA
}

(C) 2010 Wang et al; licensee BioMed Central Ltd. This is an Open Access article distributed under the terms of the Creative Commons Attribution License (http://creativecommons.org/licenses/by/2.0), which permits unrestricted use, distribution, and reproduction in any medium, provided the original work is properly cited.

[2]. Extensive studies of the tandemly repeated ribosomal RNA (rRNA) genes suggest that unequal crossover is the major driving force in the evolution of the rRNA genes with sister chromatid exchange occurring more often than exchange between homologs. Gene conversion is also believed to play a role; however, direct evidence for its involvement has not been obtained [3]. However, these mechanisms can act to achieve apparently opposite results: they can correct and eliminate new variants and they can also promote the spread of new gene variants throughout individual gene clusters, among homologous and nonhomologous chromosomes, and within an interbreeding population [4]. In recent 
years, gene conversion has become more popular in many theoretical and experimental studies [5-8]. Although gene conversion in yeast can be explained by a DNA breakage followed by invasive DNA replication [9], the molecular mechanism of gene conversion in multigene families is not well understood particularly when sequence identity is patchy [1].

Within eukaryotic genomes, rDNA gene family exists in arrays (nucleolar organizer regions or NORs on one or more chromosomes) of varying length, ranging from a single gene in the case of Tetrahymena to several thousand copies per genome in some cereals [10]. Different subunits in rDNA family are known to evolve at varying rates, depending upon the degree of selective constraint operative on each. In these subunits, the internal transcribed spacer (ITS) is not internally repetitive and its evolving rate is between those of genes and non-transcribed region [11]. Therefore, ITS is an ideal region for the study of the homogenization processes of rDNA family [12].

Natural or synthetic allopolyploids and hybrids with clear and very recent ancestry are good models for studying concerted evolution of multigene families $[6,13]$. In order to study the homogenization process of rDNA family, we exploited heterogeneity of ITS sequences (over $20 \%$ sequence divergence) in a hybrid scallop (Chlamys farreri $\$ \times$ Argopecten irradians $\sigma^{\prime}$ ). The great sequence divergence would allow the homogenization process, if any, to be readily detected in the hybrid. Here we report the homogenization of ITS sequences in the hybrid by gene conversion, leading to the generation of novel hybrid alleles. The gene conversion is massively rapid during early development of the hybrid, and is biased toward the maternal copy. We provide direct evidence for gene conversion in the ITS region of the rDNA gene clusters that may have implications not only for concerted evolution, but also for the origin of hybrid zone novel alleles, and for genetic imprinting.

\section{Results}

PCR-RFLP analysis of ITS sequences provides a rapid diagnostic tool for species identification of $C$. farreri and $A$. Irradians [14]. In the hybrid generated by crossing $C$. farreri female with $A$. irradians male, the restriction patterns using restriction enzyme Hae III differentiate the maternal ITS from paternal ITS. The $C$. farreri maternal ITS should produce three PCR-RFLP fragments of 526 bp, $117 \mathrm{bp}$ and $98 \mathrm{bp}$ (741 bp in total), while the paternal A. irradians ITS should produce three PCR-RFLP fragments of $343 \mathrm{bp}, 244 \mathrm{bp}$ and $182 \mathrm{bp}$ (769 bp in total). In order to assess dynamic variation of ITS constitution in the hybrid, PCR-RFLP was conducted at various early developmental stages. As shown in Figure 1, the proportion of the maternal and paternal alleles varied greatly with development. At the 2-cell stage, both the maternal and the paternal alleles were equally present (Figure 1A); at the trochophore stage (approximately 20 hours after fertilization), most larvae harbored alleles from both parents, while some (less than 5\%) harbored only the maternal ITS (Figure 1B); at the early stage of umbo larvae (approximately 4 days after fertilization), most larvae still harbored alleles from both parents, but signal intensities of the restriction fragments representing the paternal $A$. irradians became significantly lower in some larvae, and the proportion of the larvae that possessed only the maternal ITS allele was increased (Figure 1C); at the middle stage of umbo larvae (approximately 10 days after fertilization), most larvae possessed only the maternal ITS allele, and even in those that still possessed the parental ITS allele (less than 30\%), signal intensities of the restriction fragments representing the paternal allele of $A$. irradians were significantly lower (Figure 1D). At the late stage of umbo larvae (approximately 14 days after fertilization), the vast majority of larvae harbor only the maternal ITS allele as paternal allele was not evident when samples were analyzed individually (lanes 1 through 4, Figure 1E), although the restriction fragments from the paternal $A$. irradians allele could still be detected when multiple larvae were simultaneously included in PCR-RFLP analysis (lane 5, Figure 1E). In general, the proportion of the paternal ITS allele decreased gradually in the hybrid during the development. At the 14th day after fertilization, the paternal allele representation became extremely low (Figure 1).

Recombinant Variants and Potential Recombinant Regions: When PCR product of multiple larvae at the trochophore stage was cloned, 6 recombinant variants were identified in about 200 randomly selected colonies with PCR-RFLP technique (restriction enzymes Hae III and $M b o \mathrm{I})$. The frequencies of the six variants are $6 \%$ (RV1), 3\% (RV2), 1.5\% (RV3), 1.5\% (RV4), 1\% (RV5) and 2\% (RV6). These recombinant variants are composed of segmental sequences of C. farreri and A. irradians. These recombinant variants were probably intermediates of the paternal ITS undergoing biased gene conversion. Of the six recombinant variants, RV1, RV3, and RV4 had their first part of the amplified ITS region containing sequences from the maternal parent, and their second part of the amplified ITS region containing sequences from the paternal parent. In contrast, RV2 and RV6 contained their first part of the amplified ITS region containing sequences from the paternal parent, and the second part of the amplified ITS region containing sequences from the maternal parent. For RV5, the two terminal parts of the amplified ITS region containing sequences from the maternal parent, whereas the 


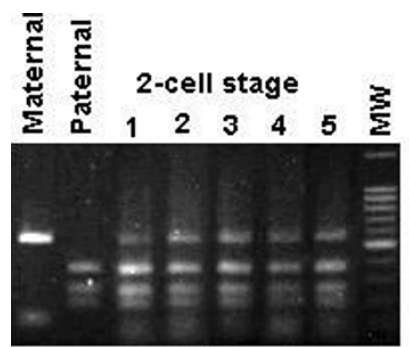

(A)

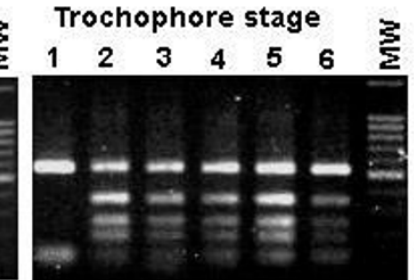

(B)

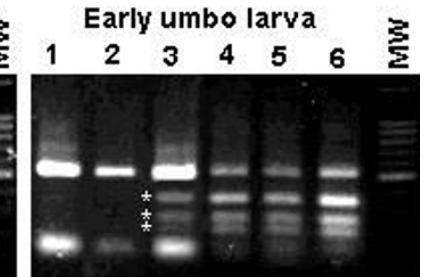

(C)

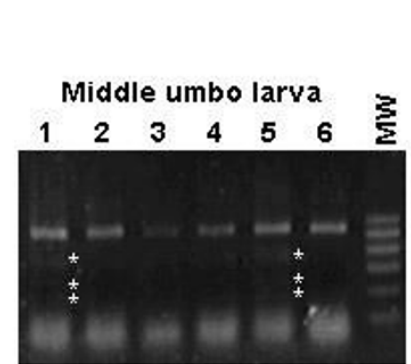

(D)

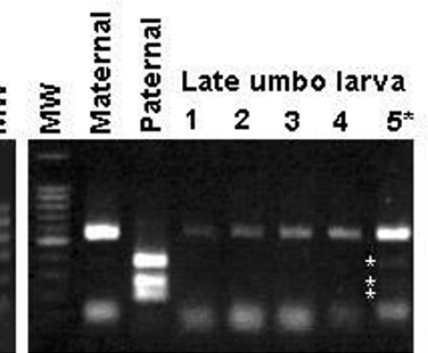

(E)

Figure 1 PCR-RFLP analysis of make-up of the ITS region in the hybrid with restriction enzyme Hae III at early development. Panel A exhibits PCR-RFLP analysis at the 2-cell stage (about 1 hour after fertilization) with samples from maternal parent C. farreri (Maternal), paternal parent A. irradians (Paternal), and samples from five random selected hybrid individuals (1 through 5), and molecular weight standard (MW). Panel B exhibits PCR-RFLP analysis at the trochophore stage (about 20 hour after fertilization) with samples from six random selected hybrid samples (1 through 6), and molecular weight standard (MW). Panel C exhibits PCR-RFLP analysis at the early umbo larva stage (about 4 days after fertilization) with samples from six random selected hybrid samples (1 through 6), and molecular weight standard (MW). Panel D exhibits PCR-RFLP analysis at the middle umbo larva stage (about 10 days after fertilization) with samples from six random selected hybrid individuals (1 through 6), and molecular weight standard (MW). Panel E exhibits PCR-RFLP analysis at the late umbo larva stage (about 14 days after fertilization) with samples from maternal parent (Maternal), paternal parent (Paternal), and samples from four random selected hybrid individuals (1 through 4) and a sample from mixed samples of multiple individuals (5*), and molecular weight standard (MW). Molecular weight standards were 100 bp DNA ladder.

middle segment of approximately 50 bp containing sequences from the paternal allele (Figure 2).

Sequence analysis revealed that all potential recombinant regions located in the regions of $5.8 \mathrm{~S}$ and ITS2 (Figure 3, for sequences involved in the recombinant regions, see Figure 2, shown in black background). These potential recombinant regions involved sequences of 9-116 bp, suggesting that recombinant variants may have resulted from site-specific recombination. Biased gene conversion in the hybrid may be drawn by site-specific recombination between the parental ITS sequences. Among these recombinant variants, RV1 and RV2 are mirror complement with exactly the same recombinant region: RV1 had its first part coming from the maternal allele and the second part coming from the paternal allele, while RV2 had the exactly opposite components with the first part coming from the paternal allele, and the second part coming from the maternal allele. This suggested that gene conversion tracts were bidirectional. However, the mirror complement of RV3-RV6 was not found, although no extensive efforts were devoted for the search and they could have also existed.
The Recombinant Variants Were Likely Amplified from Genuine Recombinant Templates: We had to consider the possibility of PCR-generated errors because all the sequenced clones were derived from PCR amplified material. While point mutations introduced by PCR as a result of infidelity of Taq polymerase are well documented, and is not the major concern in this situation, template jumping is of concern because it also can interpret the observed recombinant variants. In vitro recombination occurs when incompletely extended PCR segments from one allele serve as primer during subsequent amplification cycles amplified segments [15]. In spite of the technical difficulties in providing absolute proofs because our approaches nonetheless still involved the use of PCR, several considerations made us to believe that the observed recombinant variants were real, and were not derived from PCR artifacts. First, we conducted sequence analysis of the PCR templates for the presence of hairpins or other major secondary structures, and found no major hairpins in the observed recombinant regions except minor secondary structures with RV1, RV2 and RV3. The presumption is that 


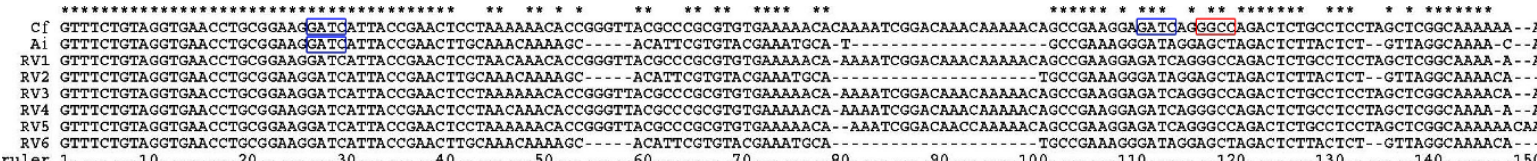

Figure 2 Alignment of parental and 6 recombinant variants' sequences. Cf: C. farreri; Ai: A. irradians; RV1-6: recombinant variants 1-6. Potential recombinant regions in recombinant variants are shown in black background. Asterisks show identical bases; dashes indicate alignment gaps. (ITS1: 34-350 bp; 5.8S: 351-506 bp; ITS2: 507-805 bp). Restriction sites for Mbo I GATC are indicated by a blue box while restriction sites for Hae III GGCC are indicated by a red box.

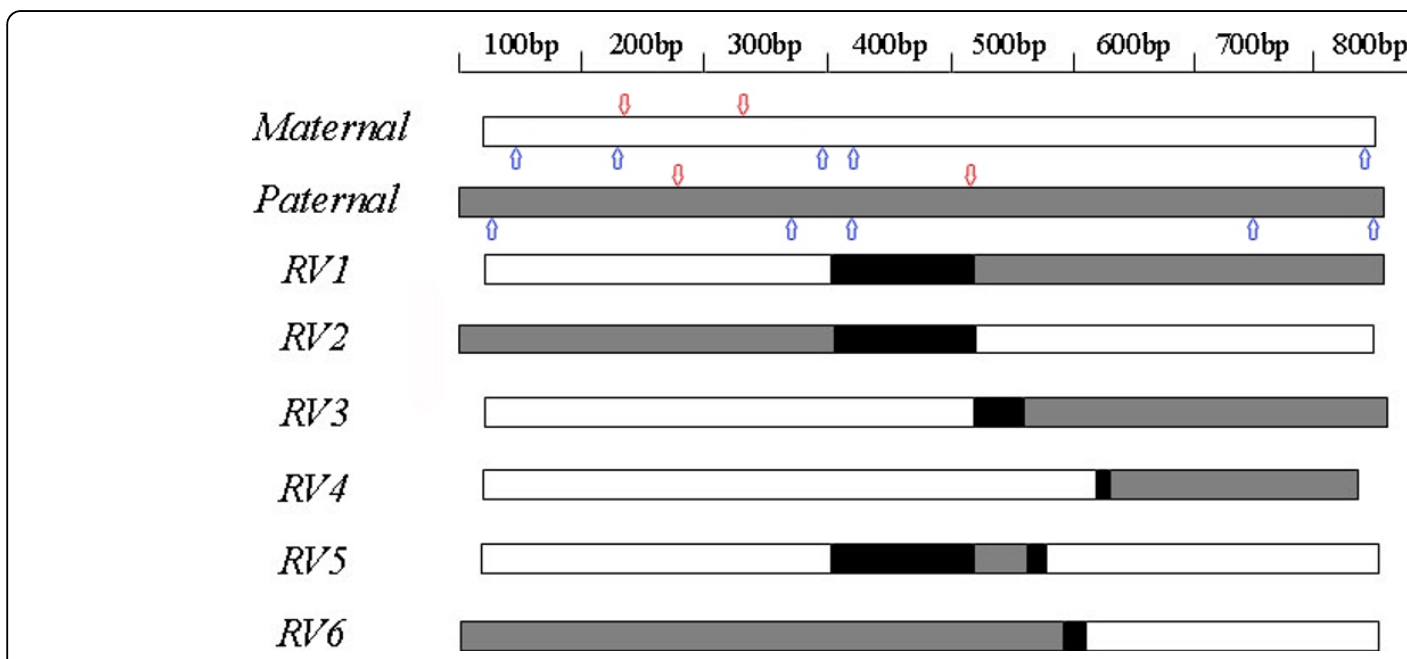

Figure 3 Schematic presentation of the recombinant variants of the interspecific hybrid showing the origins of the sequences in the ITS region. Open bar, sequences from maternal parent; sketched bar, sequences from paternal parent. Restriction sites for Mbo I are indicated by blue arrows while restriction sites for Hae III are indicated by red arrows. 
certain partially extended PCR products would have been quite abundant if they had served as primers for subsequent rounds of PCR to allow their sufficient amplification for detection using cloning with limited numbers of sequenced clones. In order to have abundant partially amplified products, secondary structure would have been required. Second, we conducted PCR directly using primers representing the recombinant variants, and PCR products were readily produced. If the recombinant variants had been absent from the template pool, success of such PCR would have required template jumping. To provide a reasonable control, we have conducted PCR amplification using a mixture of DNA isolated from maternal C. farreri and paternal A. irradians as templates. No PCR products were generated from such mixed templates, suggesting that PCR templates for the recombinant variants were genuinely present in the hybrid scallops. Third, we considered mathematical assessment of product abundance. In this regard, all the recombinant variants but RV5 requires just one recombination while RV5 requires two recombination, and they were all readily detectable from limited sequenced clones. If indeed template jumping was involved, molecules requires more than one recombination should be very rare as it can be picked up several rounds later in subsequent PCR cycles. In addition, the molar ratio of the partially extended products to the PCR primers would be miniscule, and annealing of long, partially extended products would otherwise require more time than the annealing of short PCR primers. This consideration, however, is just theoretical as opposite conclusions were reached by Bradley and Hillis with $29 \%$ each of the parental types and $43 \%$ of recombinant variants, several of which required more than one recombination (see discussions below).

Cellular Evidence for Maternal Biased Gene Conversion of ITS Alleles: Two competing hypotheses can explain the reduction of the paternal ITS allele in the hybrid: (i) the rDNA-containing chromosomes of $A$. irradians have been gradually lost in the hybrid, since interspecific hybridization is commonly accompanied by chromosome expulsion, or (ii) biased gene conversion which makes the paternal ITS be gradually converted to the maternal ITS, occurred in rDNA family of the hybrid. In order to differentiate the two possibilities, fluorescent in situ hybridization (FISH) and genomic in situ hybridization (GISH) were conducted to determine the status of ITS on chromosomes (Figure 4). It has been reported that rDNA family of $C$. farreri is solely located on the telomeric region of the short arm of

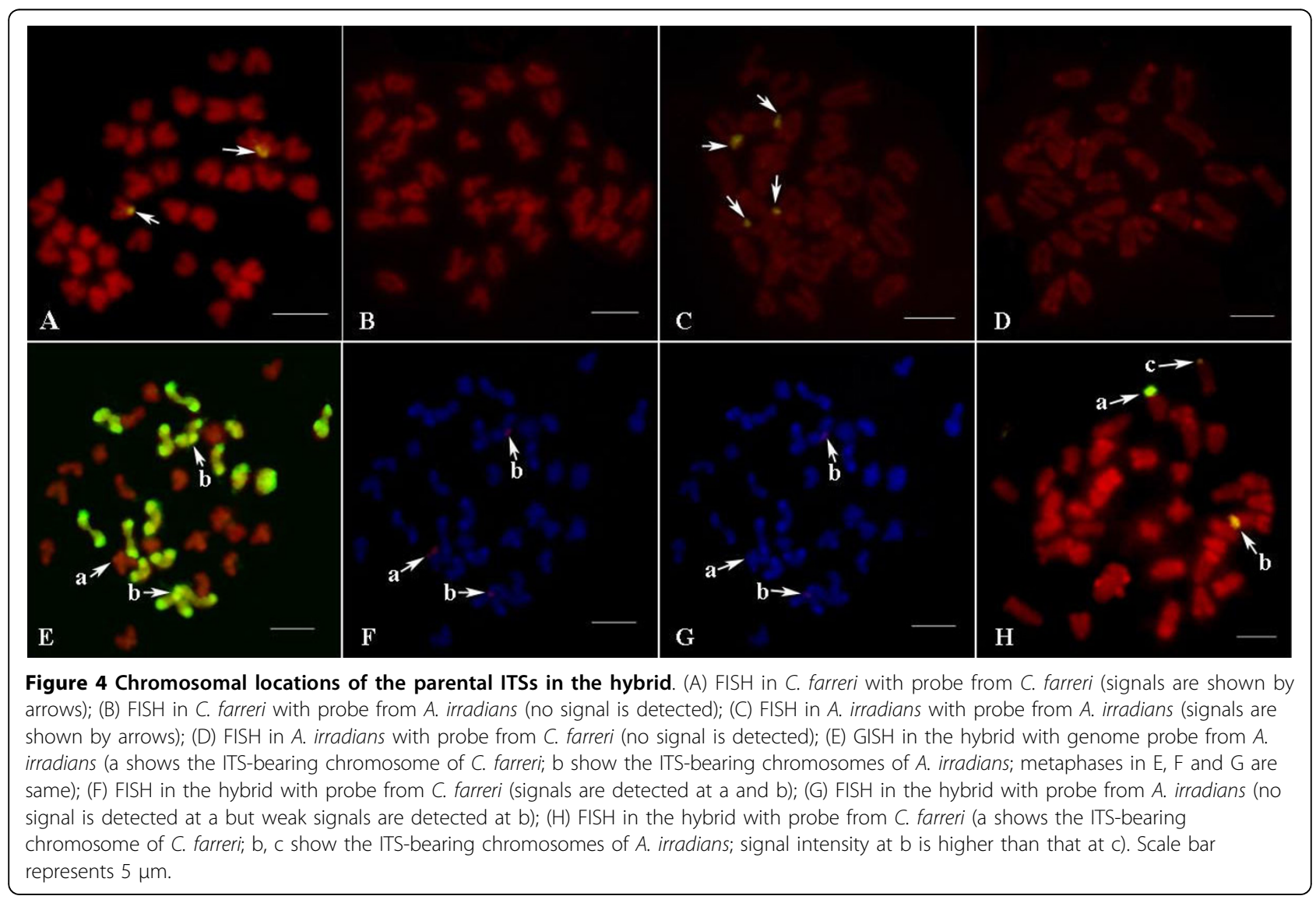


chromosome 5, while that of A. irradians is located on the telomeric region of the short arm of chromosome 4 and chromosome 8 [16]. Because of the significant levels of sequence divergence, (sequence homogeneity less than $80 \%$ ), the parental ITS sequences can be distinguished by FISH technique under the optimized condition, which allowed us to gain direct cellular evidence for the biased gene conversion.

Chromosomal lose was not involved in the observed reduction of the paternal allele. GISH analysis revealed that the haploid chromosomes from both parents were intact in the hybrid in more than $66 \%$ metaphases (see Fig. 4E). In FISH analysis, when probe from C. farreri was used for hybridization with the hybrid chromosomes, one signal was found in the ITS-bearing chromosome of $C$. farreri as expected, while two signals were found in the ITS-bearing chromosomes of A. irradians (see Fig. 4F), suggesting the presence of $C$. farreri ITS allele sequences on the ITS-bearing chromosomes of $A$. irradians. Moreover, intensities of the two signals that found in the ITS-bearing chromosomes of $A$. irradians were significantly inconsistent in some metaphases (see Fig. $4 \mathrm{H}$ ). When probe from A. irradians was used, only two weak signals were found in the ITS-bearing chromosomes of $A$. irradians as expected, while no signal was found in the ITS-bearing chromosome of $C$. farreri (see Fig. 2G). Taken together, these results provide cellular evidence for the maternal biased gene conversion at the ITS region of the rDNA loci, which confirm the molecular evidence for maternal biased gene conversion.

\section{Discussion}

This work took advantage of the hybrid from two scallop species C. farreri and A. irradians, whose ITS sequences are readily distinguishable, for the study of concerted sequence homogenization of the rDNA. As reviewed by Eickbush and Eickbush [3], unequal crossover has been regarded as the major mechanism for the evolution of rRNA genes. Here, we provided strong molecular and cellular evidence for maternal biased gene conversion, leading to reduction of the paternal allele toward homogenization with the maternal allele. Although unequal crossover remains a possibility, it does not support to explain the observed sequence homogenization. First, it is difficult to explain why paternal ITS sequences can be totally replaced just through mitotic cell divisions by unequal crossover. Second, about $70 \%$ C. farreri eggs can be fertilized with $A$. irradians sperm. About $80 \%$ fertilized eggs can be developed to trochophore larvae, which is comparable to that observed in the intra-specific cross of C. farreri. We used trochophore larvae at the early stage for chromosome spread preparation, and yet, no signal was observed on the maternal rRNA loci using paternal sequence as the probe in the FISH experiment, which suggests unequal crossover did not occur between paternal and maternal rRNA loci-containing chromosomes. Although most theoretical and experimental studies on concerted evolution of rDNA family were conducted in model organisms such as yeast, Drosophila, and mice as well as in humans [1], non-traditional model system can provide cleaner evidence for the mechanisms involved in sequence homogenization, as demonstrated here with the scallops. In this paper, maternal biased conversion of ITS sequences was evident during early stages of development of the hybrid scallop. Hybrid larvae at later developmental stages have not been obtained due to high mortality rate, and are therefore not included in the current study.

\section{Site-specific recombination and double-strand breaks} (DSBs)

In yeast, DSBs are considered as the sole mechanism of recombination in meiotic cells and are a major factor in recombination in mitotic cells [9]. In a general model, DSBs are cut on both strands to create large gaps flanked by rather short regions of single-stranded DNA that could invade a homologous template and initiate DNA repair[17]. In this study, potential recombinant regions identified in the hybrid are also conservative in the ITS sequences of other 6 species of Pectinidae which represent all now available in GenBank. Recombination in these regions may be site-specific but not sequence-specific since common sequences of these regions are not found. Some sites in these regions may be recognized by some endonucleases, which may be analogous to Spo11p in Saccharomyces cerevisiae $[18,19]$, to generate DSBs. In general, homologous recombination in DSB repair involves hundreds of nearly perfectly matched base pairs. However, most recombinant regions identified in this study are very short. It is not unique since homologous recombination in DSB repair can also occur with surprisingly short homologous regions in yeast [9]. Moreover, conversion tracts in RV3-RV6 could be unidirectional because the counterparts of these recombinant variants were not found. The phenomenon that conversion tracts extended only on one side of the DSB was also reported in yeast in meiosis and mitosis [20-22].

Although identification of recombination variants have also been reported in ITS1 sequences of Darwinula stevensoni [23], there still exists the possibility that recombinant variants are artifacts generated by PCR amplification [15]. In order to exclude this possibility, PCR product amplified from the mixture of genomic DNA of C. farreri and A. irradians was cloned and recombination variants were subsequently screened in 300 randomly selected colonies. Only RV1 and RV2 were identified at significantly lower frequency, which 
suggests that short homologous regions in RV3-RV6 may be not significant to introduce recombination in PCR amplification. Moreover, as an alternative technique, loop-mediated isothermal amplification (LAMP) developed by Notomi et al. [24] was also used in this study (data not shown). Because cycles of denaturation in PCR amplification are not needed in this technique, this technique can significantly avoid the generation of recombinant variants. When specific primers were designed for RV6 (FIP: 5'-ACAGCCGACCCTCAGACACATCGATATCTTGAACGCACA

TTGC-3'; BIP: 5'-CCGGCGAGCGGTCTTAAACTGGTTTGTTTTTGGTTCGATTG

GA-3'; F3: 5'-TGTGAATTGCAGGACACATTGA-3'; B3: 5'-GCGTCTCTTGTAATTT

GTTCCGTA-3'), RV6 could be amplified from the hybrid and further was confirmed by subsequent sequencing.

Biased gene conversion: Gene conversion can be bidirectional as those reported in Gossypium allopolyploid and Nicotiana allopolyploid $[6,25]$. However, gene conversion is biased in the hybrid in this study and similar phenomenon was also reported in other studies [26-28]. This phenomenon may be general in Pectinidae, because biased gene conversion was also observed in other hybrids such as $C$. farreri $\$ \times$ Chlamys nobilis $0^{\prime \prime}, C$. farreri $\bigcirc \times$ Patinopecten yessoensis $\sigma^{\prime \prime}$, and $P$. yessoensis $\$ \times$ A. irradians $\sigma^{\text {" }}$ (our unpublished data).

The possible mechanism for biased gene conversion in the hybrid may involve maternal restriction systems. In the initial formation of the hybrid, maternal enzymatic system may treat the chromosomes of $A$. irradians as intruding chromosomes. Specific sites in paternal recombinant regions are thus recognized by maternal endonucleases to produce DSBs. Biased gene conversion can subsequently occur when the directionality of DSB repair is in favor of the maternal DNA sequences. There were evidences from yeast that the directionality of DSB repair is in favor of the donor sequences $[29,30]$.

\section{The rate of gene conversion and mitosis}

Although gene conversion was almost complete in all individuals sampled at the late stage of umbo larvae (about 14 days after fertilization), this process could have started well within 20 hours after fertilization (see Fig. 1). The rate of gene conversion is surprisingly rapid in the hybrid. For rDNA family, although the highest rate calculated from hybrids of Armeria was one order of magnitude above that in Drosophila reported by Dover [31], one generation is still needed [26]. Liao et al. [32] demonstrated that it is even possible that concerted evolution could be achieved quite rapidly, perhaps within one or a few meioses or mitoses. Because of absence of meiosis, the process of gene conversion in this study thus may occur in mitosis. Rapid gene conversion may be involved with long conversion tracts and/or at high frequency. In mitosis, conversion tracts in yeast could even extend up to $400 \mathrm{~kb}[9,33]$. In general, exchange rates between rDNA arrays residing in the heterochromatin were expected to be rather low. However, it was found that recombination between the rDNA arrays occurs at a much higher rate than the rest regions of the heterochromatin in Drosophila melanogaster [34]. It has been suggested in some studies that gathering of rDNA in/at the nucleolus and its exposure to nucleolin play an important role in recombination [35-37].

\section{Gene conversion between non-homologous chromosomes}

It is currently unclear that which of rDNA-containing chromosomes of $A$. irradians is homologous to that of C. farreri. Inconsistent numbers of rDNA locus in the two species suggest that at least one of rDNA-containing chromosomes of $A$. irradians is nonhomologous to that of $C$. farreri. Based on the result that two signals were found in the ITS-bearing chromosomes of A. irradians in the hybrid when probe from $C$. farreri was used, biased gene conversion had occurred between nonhomologous chromosomes as well as between the homologous chromosomes in the hybrid. Recombination between nonhomologous chromosomes may be more convenient in the hybrid since rDNA loci in the parents are all located on the telomeric region of short arm of chromosomes. Gene conversion among rDNA loci on nonhomologous chromosomes were also reported in humans and apes [38,39]. In the hybrid, intensities of two signals were found significantly inconsistent in some metaphases, which may imply that the rates of gene conversion are different between homologous chromosomes and nonhomologous chromosomes. For example, in Fig. $2 \mathrm{H}, a$ may be more homologous to $b$ than to $c$, because signal intensity in $b$ is higher than that in $c$.

The maternally biased gene conversion reported here may have important implications for concerted gene evolution among gene families, as the similar mechanism can be used for sequence homogenization in various multigene families. The appearances of recombinant variants could help explain the origin of hybrid zone novel alleles, a phenomenon long puzzled the scientific community [40]. In spite of their interpretation of recombinant variants as being generated from PCR template jumping [15], the high proportion of recombinant (43\%) may argue against their conclusion. In our case here, although the frequency of recombinant variants was relatively low, their evolutionary consequences can be highly significant. If such a mechanism is involved in hybrids, or crossbreeds of more distantly related populations, its impact on the emergence of novel alleles 
would be tremendously high. The biased gene conversion would also have significance implications for genetic imprinting where only one allele from a specific parental origin is expressed. Therefore, the hybrid scallop offers a unique system for further studies involving all of these issues.

\section{Conclusions}

Taken together, these molecular and cellular evidences support rapid concerted gene evolution via maternally biased gene conversion. As such a process would lead to the expression of only one parental genotype, and have the opportunities to generate recombinant intermediates; this work may also have implications in novel hybrid zone alleles and genetic imprinting, as well as in concerted gene evolution. In the course of evolution, many species may have evolved involving some levels of hybridization, intra- or interspecific, the sex-biased sequence homogenization could have led to a greater role of one sex than the other in some species.

\section{Methods}

\section{Scallop Materials}

Several sex-matured individuals of C. farreri and A. irradians were obtained from an aquacultural hatchery in Penglai, Shandong Province, China. Artificial hybridization $\left(C\right.$. farreri $\$ \times A$. irradians $\left.\sigma^{\prime}\right)$ was carried out in a laboratory. Because A. irradians is hermaphroditic, sperm was filtered by a sieve cloth (500 meshes) in order to avoid introducing eggs of $A$. irradians. After fertilization, hybrid larvae were reared at $20^{\circ} \mathrm{C}$. Samples of hybrid larvae were taken at the 2-cell stage (about 1 hour after fertilization), the trochophore stage (about 20 hours after fertilization), the early stage of umbo larvae (about 4 days after fertilization), the middle stage of umbo larvae (about 10 days after fertilization) and the late stage of umbo larvae (about 14 days after fertilization). The muscles of parental scallops were preserved at $-20^{\circ} \mathrm{C}$ and the larvae were stored in ethanol at $4^{\circ} \mathrm{C}$.

\section{DNA Extraction}

Genomic DNA of parental scallops was extracted from frozen muscle tissues with phenol/chloroform extraction as described by Sambrook et al [41]. Larval DNA extraction was described as follows. Larvae were transferred from ethanol to a cavity slide and left until the ethanol evaporated completely. Then the larvae were rinsed and agitated with sterile pure water. In total, 30 larvae at each stage were individually isolated through micro-operation and then transferred on a mounted needle to a $0.2 \mathrm{~mL}$ PCR tube containing $10 \mu \mathrm{L}$ of STE solution $(100 \mathrm{mM}$ $\mathrm{NaCl} ; 10 \mathrm{mM}$ Tris-Cl [pH 8.0]; 1 mM EDTA [pH 8.0]; 0.5 $\mathrm{mg} / \mathrm{mL}$ proteinase $\mathrm{K}$ ). The tubes were kept at $56^{\circ} \mathrm{C}$ for 30 min to break the cells and expose the DNA, and then $95^{\circ}$ $\mathrm{C}$ for $10 \mathrm{~min}$ to inactivate proteinase $\mathrm{K}$.

\section{PCR Amplification}

PCR amplifications were set up in a $20 \mu \mathrm{L}$ volume composed of $100 \mathrm{ng}$ parental genomic DNA or $10 \mu \mathrm{L}$ of larval DNA solution, $0.2 \mu \mathrm{M}$ each primer, $2 \mathrm{mM} \mathrm{MgCl}$, $0.2 \mathrm{mM}$ each dNTP, $1 \times$ PCR reaction buffer, and $1 \mathrm{U}$ Taq polymerase (Promega Inc., Shanghai, China). According to the primers designed for Mytilus mussels [42], a pair of primers (forward: 5' GTTTCTGTAGGTGAACCTG 3'; reverse: 5' CTCGTCTGATCTGAGGTCGGA 3') were used in this study. These anneal at the $3^{\prime}$ end of $18 \mathrm{~S}$ rRNA gene and the 5 ' end of 28S rRNA gene, amplifying ITS1, 5.8S gene and ITS2. Thermal cycling used a PTC-100 cycler (MJ Research Inc., USA). All PCR cycles began with an initial denaturation at $94^{\circ} \mathrm{C}$ for 3 minutes, followed by 30 cycles of $94^{\circ} \mathrm{C} 30 \mathrm{~s}, 54^{\circ} \mathrm{C} 30 \mathrm{~s}$, and $72^{\circ} \mathrm{C} 1 \mathrm{~min}$, and a final extension at $72^{\circ} \mathrm{C}$ for $10 \mathrm{~min}$.

\section{PCR-RFLP Analysis}

Two restriction enzymes, Hae III and Mbo I, were used in the PCR-RFLP analysis. Restriction digestions were performed in $10 \mu \mathrm{l}$ volumes, containing $3 \mu \mathrm{l}$ of PCR product, $2 \mathrm{U}$ of restriction enzyme and $1 \mu \mathrm{l}$ buffer supplied by the manufacturer (NEB Inc., USA). The reaction was incubated at $37^{\circ} \mathrm{C}$ for $6 \mathrm{~h}$ and then stopped by inactivating the restriction enzyme at $80^{\circ} \mathrm{C}$ for $20 \mathrm{~min}$. Restriction digestion products were analyzed by gel electrophoresis through an agarose gel (1.0\%).

\section{Cloning, Sequencing and Sequence Analysis}

PCR product of multiple larvae at the trochophore stage was ligated into pMD18-T (Takara Inc., Dalian, China) and subsequently transformed into Escherichia coli DH5 $\alpha$ cells. Using PCR-RFLP technique, recombinant variants were screened in 500 randomly selected colonies and corresponding colonies were sequenced with a 3730 automatic sequencer (Applied Biosystems Inc., USA). Alignment of the parental and recombinant variants' sequences was performed using the program ClustalX 1.83[43].

\section{Chromosome Preparations, FISH and GISH Analysis}

Following treatment with colchicine $(0.01 \%)$ for $2 \mathrm{~h}$ at room temperature (RT), trochophore were exposed to $0.075 \mathrm{M} \mathrm{KCl}$ solution for $30 \mathrm{~min}$ and then fixed three times (15 min each) in fresh ethyl alcohol/glacial acetic acid solution (3:1). After being treated with $50 \%$ acetic acid, the fixed larvae were dissociated into a cell suspension, and then dropped onto hot-wet slides and airdried.

In FISH experiments, probes were labeled by PCR with biotin-16-dUTP. Chromosome spreads were pretreated with $100 \mu \mathrm{g} / \mathrm{ml}$ DNase-free RNase A in $2 \times$ SSC for $1 \mathrm{~h}$ at $37^{\circ} \mathrm{C}$, and then treated with $0.005 \%$ pepsin in $10 \mathrm{mM} \mathrm{HCl}$ for $10 \mathrm{~min}$ at $37^{\circ} \mathrm{C}$. Chromosome preparations were denatured in a mixture containing $70 \%$ formamide and $2 \times \mathrm{SSC}$ at $72^{\circ} \mathrm{C}$ for $2 \mathrm{~min}$, dehydrated 
with a chilled ethanol series $(70 \%, 90 \%$, and $100 \%$; 5 min each), and then air-dried. Slides were then incubated with $20 \mu \mathrm{l}$ of denatured hybridization mix $(5 \mathrm{ng} / \mu \mathrm{l}$ probe, $10 \%$ dextran sulphate, $250 \mathrm{ng} / \mu \mathrm{l}$ salmon sperm DNA, $50 \%$ deionized formamide in $2 \times \mathrm{SSC}, 80^{\circ} \mathrm{C}$ for 5 min and cooled immediately) for $16 \mathrm{~h}$ at $37^{\circ} \mathrm{C}$ in a moist chamber. After hybridization, slides were washed three times ( $5 \mathrm{~min}$ each) in $50 \%$ formamide in $2 \times \mathrm{SSC}$ at $66^{\circ} \mathrm{C}$, three times ( $5 \mathrm{~min}$ each) in $1 \times \mathrm{SSC}$ at $66^{\circ} \mathrm{C}$, once for $5 \mathrm{~min}$ in $2 \times \mathrm{SSC}$ at room temperature. Hybridized probes were detected with fluorescein-labeled avidin DCS (Vector Laboratories). Chromosomes were counterstained with $1.5 \mu \mathrm{g} / \mathrm{ml}$ propidium iodide (PI) or 4'-6-Diamidino-2-phenylindole (DAPI) in antifade solution (Vector Laboratories). For multiple hybridizations, slides were washed three times by $2 \times$ SSC to remove PI or DAPI before denaturation in the subsequent hybridization. Slides were observed using a Nikon Eclipse-600 epifluorescence microscope equipped with a CCD camera. The signals were collected using appropriate filter sets and LUCIA software (Laboratory Imaging).

In GISH experiments, genomic DNA from A. irradians was used as the template for labeling a probe. The probe was labeled by nick translation with biotin-16dUTP. All steps in GISH experiments were the same as described for FISH experiments except that the temperature of post-hybridization wash was set as $42^{\circ} \mathrm{C}$.

\section{Acknowledgements}

The authors thank Prof. Guanpin Yang for reviewing this manuscript. This work is supported by grants from '863' Hi-Tech Research and Development Program of China (No. 2006AA10A408 and No. 2006AA10A402) and National Nonprofit Special Grant of China (No. nyhyzx07-047) and by the Key Laboratory of Mariculture of Ministry of Education, Ocean University of China (200417).

\section{Author details}

${ }^{1}$ Key Laboratory of Marine Genetics and Breeding of Ministry of Education, Ocean University of China, Qingdao 266003, China. ${ }^{2}$ The Fish Molecular Genetics and Biotechnology Laboratory, Aquatic Genomics Unit, Department of Fisheries and Allied Aquacultures and Program of Cell and Molecular Biosciences, Auburn University, Auburn, AL 36849, USA.

\section{Authors' contributions}

SW and ZB designed the study. SW and LZ conducted the experiment. SW, $J H, Z B$ and $Z L$ conducted data analysis. ZB supervised the entire study. SW, $Z B$ and $Z L$ wrote the manuscript. All authors read and approved the final manuscript.

\section{Received: 17 July 2009}

Accepted: 11 January 2010 Published: 11 January 2010

\section{References}

1. Nei M, Rooney AP: Concerted and birth-and-death evolution of multigene families. Annu Rev Genet 2005, 39:121-152.

2. Dover G: Molecular drive: a cohesive mode of species evolution. Nature 1982, 299(5879):111-117.

3. Eickbush TH, Eickbush DG: Finely orchestrated movements: evolution of the ribosomal RNA genes. Genetics 2007, 175:477-85.
4. Gonzalez IL, Sylvester JE: Human rDNA: evolutionary patterns within the genes and tandem arrays derived from multiple chromosomes. Genomics 2001, 73(3):255-263.

5. Hughes $\mathrm{KW}$, Petersen $\mathrm{RH}$ : Apparent recombination or gene conversion in the ribosomal ITS region of a Flammulina (Fungi, Agaricales) hybrid. Mol Biol Evol 2001, 18(1):94-96.

6. Kovarik A, Matyasek R, Lim KY, Skalick K, Koukalov B, Knapp S, Chase M, Leitch AR: Concerted evolution of $185.826 \mathrm{~S}$ rDNA repeats in Nicotiana allotetraploids. Biological Journal of the Linnean Society 2004, 82:615-625.

7. Parkin EJ, Butlin RK: Within- and between-individual sequence variation among ITS1 copies in the meadow grasshopper Chorthippus parallelus indicates frequent intrachromosomal gene conversion. Mol Biol Evol 2004, 21(8):1595-1601.

8. Santoyo G, Romero D: Gene conversion and concerted evolution in bacterial genomes. FEMS Microbiol Rev 2005, 29(2):169-183.

9. Paques F, Haber JE: Multiple pathways of recombination induced by double-strand breaks in Saccharomyces cerevisiae. Microbiol Mol Biol Rev 1999, 63(2):349-404.

10. Elder JF Jr, Turner BJ: Concerted evolution of repetitive DNA sequences in eukaryotes. Q Rev Biol 1995, 70(3):297-320.

11. Lopez-Pinon MJ, Insua A, Mendez J: Identification of four scallop species using PCR and restriction analysis of the ribosomal DNA internal transcribed spacer region. Mar Biotechnol (NY) 2002, 4(5):495-502.

12. Schlotterer C, Tautz D: Chromosomal homogeneity of Drosophila ribosomal DNA arrays suggests intrachromosomal exchanges drive concerted evolution. Curr Biol 1994, 4(9):777-783.

13. Kovarik A, Pires JC, Leitch AR, Lim KY, Sherwood AM, Matyasek R, Rocca J, Soltis DE, Soltis PS: Rapid concerted evolution of nuclear ribosomal DNA in two Tragopogon allopolyploids of recent and recurrent origin. Genetics 2005, 169(2):931-944

14. Wang S, Bao Z, Zhang L, Li N, Zhan A, Guo W, Wang L, Hu J: A new strategy for species identification of planktonic larvae: PCR-RFLP analysis of the internal transcribed spacer region of ribosomal DNA detected by agarose gel electrophoresis or DHPLC. J Plankton Res 2006, 28:375-384.

15. Bradley RD, Hillis DM: Recombinant DNA sequences generated by PCR amplification. Mol Biol Evol 1997, 14(5):592-593.

16. Wang $Y$, Guo $X$ : Chromosomal rearrangement in pectinidae revealed by rRNA loci and implications for bivalve evolution. Biol Bull 2004, 207(3):247-256.

17. Szostak JW, Orr-Weaver TL, Rothstein RJ, Stahl FW: The double-strandbreak repair model for recombination. Cell 1983, 33(1):25-35.

18. Bergerat A, de Massy B, Gadelle D, Varoutas PC, Nicolas A, Forterre P: An atypical topoisomerase II from Archaea with implications for meiotic recombination. Nature 1997, 386(6623):414-417.

19. Keeney S, Giroux CN, Kleckner N: Meiosis-specific DNA double-strand breaks are catalyzed by Spo11, a member of a widely conserved protein family. Cell 1997, 88(3):375-384.

20. Nelson HH, Sweetser DB, Nickoloff JA: Effects of terminal nonhomology and homeology on double-strand-break-induced gene conversion tract directionality. Mol Cell Biol 1996, 16(6):2951-2957.

21. Porter SE, White MA, Petes TD: Genetic evidence that the meiotic recombination hotspot at the HIS4 locus of Saccharomyces cerevisiae does not represent a site for a symmetrically processed double-strand break. Genetics 1993, 134(1):5-19.

22. Sweetser DB, Hough H, Whelden JF, Arbuckle M, Nickoloff JA: Fineresolution mapping of spontaneous and double-strand break-induced gene conversion tracts in Saccharomyces cerevisiae reveals reversible mitotic conversion polarity. Mol Cell Biol 1994, 14(6):3863-3875.

23. Gandolfi A, Bonilauri P, Rossi $V$, Menozzi P: Intraindividual and intraspecies variability of ITS1 sequences in the ancient asexual Darwinula stevensoni (Crustacea: Ostracoda). Heredity 2001, 87(Pt 4):449-455.

24. Notomi T, Okayama H, Masubuchi H, Yonekawa T, Watanabe K, Amino N, Hase T: Loop-mediated isothermal amplification of DNA. Nucleic Acids Res 2000, 28(12):E63.

25. Wendel JF, Schnabel A, Seelanan T: Bidirectional interlocus concerted evolution following allopolyploid speciation in cotton (Gossypium). Proc Natl Acad Sci USA 1995, 92(1):280-284.

26. Fuertes Aguilar J, Rossello JA, Nieto Feliner G: Nuclear ribosomal DNA (nrDNA) concerted evolution in natural and artificial hybrids of Armeria (Plumbaginaceae). Mol Ecol 1999, 8(8):1341-1346. 
27. Ganley AR, Scott B: Concerted evolution in the ribosomal RNA genes of an Epichloe endophyte hybrid: comparison between tandemly arranged rDNA and dispersed 5 S rrn genes. Fungal Genet Biol 2002, 35(1):39-51.

28. Hillis DM, Moritz C, Porter CA, Baker RJ: Evidence for biased gene conversion in concerted evolution of ribosomal DNA. Science 1991, 251(4991):308-310.

29. Leung W, Malkova A, Haber JE: Gene targeting by linear duplex DNA frequently occurs by assimilation of a single strand that is subject to preferential mismatch correction. Proc Natl Acad Sci USA 1997, 94(13):6851-6856.

30. Ray BL, White $\mathrm{Cl}$, Haber JE: Heteroduplex formation and mismatch repair of the "stuck" mutation during mating-type switching in Saccharomyces cerevisiae. Mol Cell Biol 1991, 11(10):5372-5380.

31. Dover GA: Linkage disequilibrium and molecular drive in the rDNA gene family. Genetics 1989, 122(1):249-252.

32. Liao D, Pavelitz T, Kidd JR, Kidd KK, Weiner AM: Concerted evolution of the tandemly repeated genes encoding human U2 snRNA (the RNU2 locus) involves rapid intrachromosomal homogenization and rare interchromosomal gene conversion. EMBO J 1997, 16(3):588-598.

33. Esposito MS: Evidence that spontaneous mitotic recombination occurs at the two-strand stage. Proc Natl Acad Sci USA 1978, 75(9):4436-4440.

34. Williams SM, Kennison JA, Robbins LG, Strobeck C: Reciprocal recombination and the evolution of the ribosomal gene family of Drosophila melanogaster. Genetics 1989, 122(3):617-624.

35. Borggrefe $T$, Wabl M, Akhmedov AT, Jessberger R: A B-cell-specific DNA recombination complex. J Biol Chem 1998, 273(27):17025-17035.

36. Hanakahi LA, Dempsey LA, Li MJ, Maizels N: Nucleolin is one component of the $B$ cell-specific transcription factor and switch region binding protein, LR1. Proc Natl Acad Sci USA 1997, 94(8):3605-3610.

37. Thyagarajan B, Lundberg R, Rafferty M, Campbell C: Nucleolin promotes homologous DNA pairing in vitro. Somat Cell Mol Genet 1998, 24(5):263272.

38. Arnheim N, Krystal M, Schmickel R, Wilson G, Ryder O, Zimmer E: Molecular evidence for genetic exchanges among ribosomal genes on nonhomologous chromosomes in man and apes. Proc Natl Acad Sci USA 1980, 77(12):7323-7327

39. Krystal M, D'Eustachio P, Ruddle FH, Arnheim N: Human nucleolus organizers on nonhomologous chromosomes can share the same ribosomal gene variants. Proc Natl Acad Sci USA 1981, 78(9):5744-5748.

40. Bradley RD, Bull JJ, Johnson AD, Hillis DM: Origin of a novel allele in a mammalian hybrid zone. Proc Natl Acad Sci USA 1993, 90(19):8939-8941.

41. Sambrook J, Fritsch E, Maniatis T: Molecular cloning: a laboratory manual. Cold Springs Harbor Laboratory Press, New York 1989, 464-467.

42. Heath D, Rawson P, Hilbish T: PCR-based nuclear markers identify alien blue mussel (Mytilus spp.) genotypes on the west coast of Canada. Can J Fish Aquat Sci 1995, 52:2621-2627.

43. Thompson JD, Gibson TJ, Plewniak F, Jeanmougin F, Higgins DG: The CLUSTAL_X windows interface: flexible strategies for multiple sequence alignment aided by quality analysis tools. Nucleic Acids Res 1997, 25(24):4876-4882

doi:10.1186/1471-2148-10-6

Cite this article as: Wang et al: Molecular and cellular evidence for biased mitotic gene conversion in hybrid scallop. BMC Evolutionary Biology 2010 10:6.

Publish with Bio Med Central and every scientist can read your work free of charge

"BioMed Central will be the most significant development for disseminating the results of biomedical research in our lifetime. "

Sir Paul Nurse, Cancer Research UK

Your research papers will be:

- available free of charge to the entire biomedical community

- peer reviewed and published immediately upon acceptance

- cited in PubMed and archived on PubMed Central

- yours - you keep the copyright 